\title{
Network Life Time maximization of the AOMDV Protocol Using Nodes Energy Variation
}

\author{
Amir Abdelkader AOUIZ ${ }^{1,2}$, Sofiane BOUKLI HACENE ${ }^{1}$ \\ Pascal LORENZ ${ }^{2}$, Marc GILG ${ }^{2}$ \\ ${ }^{1}$ EEDIS Laboratory , computer science departement, Djillali Liabes University, 22000, \\ Sidi Bel Abbes (Alegria) \\ ${ }^{2}$ University of Haute Alsace, 68000, Colmar (France) \\ E-mail: amir-abdelkader.aouiz@uha.fr, boukli@gmail.com, pascalorenz@gmail.com, \\ marc.gilg@uha.fr
}

Received: April 1, 2018

Accepted: June 27, 2018

Published: June 29, 2018

DOI: 10.5296/npa.v10i2.13322

URL: https://doi.org/10.5296/npa.v10i2.13322

\begin{abstract}
Mobile ad hoc network presents generally several challenges such as high dynamic topology, packet loss and frequent routes discovering that result in low throughput and reduced packet delivery ratio. To reduce the number of route discovering process, multipath routing protocols makes use of alternate paths to continue packets transmission. Ad Hoc On-demand, multipath distance vector routing protocol or AOMDV, is one of the well-known multipath protocols that relays on the hop count metric to route packets. However maintaining disjoint active routes can reduce nodes life time and lead to more control messages like error and discovering packets. In this paper, a pre-emptive approach is proposed based on the historical values of nodes energy. Nodes that excessively consume energy in time are considered overloaded and are excluded from route discovering, hence nodes with reduced energy variation are selected to route packets. Simulation results show reduced number of dead nodes by $30 \%$, overhead by $16 \%$.
\end{abstract}

Keywords: MANET, AOMDV Routing Protocol, Alternate Paths, Energy Variation Rate, Network Traffic History, Quality of Service. 


\section{Introduction}

A mobile ad hoc network or MANET is a wireless communication network formed by cooperating mobile nodes to deliver packets. It presents several challenges such as high dynamic topology, frequent nodes movement change, low power capacity and limited throughput and bandwidth [1]. In such network scenarios, it is very important to manage the packets routing effectively with the minimum of time and effort. Therefore, the routing protocol must be adapted to the frequent topological changes in the fastest possible way.

The -on-demand routing protocols provide the opportunity to discover and maintain a route whenever there is a need for it. Several reactive protocols have been proposed and implemented, these includes the "Dynamic Source Routing" (DSR) and the "Ad hoc On-Demand Distance Vector" (AODV) [2]. The AODV is the most common reactive routing protocol for MANETs. Although the AODV provides a good performance and latency in small networks size, it incurs a high overhead when the network size increases, this may lead to a degradation of the protocol performance [3].

In fact, during route discovery process, multiple paths might be discovered. The source node chooses the best path among them to route its packets, the rest of paths are discarded. However, even best paths might break, and since there is no alternate path to rely on it, it leaves no choice to intermediate nodes but to drop their current packets [4]. This packet loss may result in lower throughput and reduced packet delivery ratio. In addition, in high dynamic topology, the cost of a new route discovery in term of latency and overhead is too high; the frequent routes discovering process can increase significantly the average end-to-end delay [5].

The Multipath on-demand protocols have great ability to reduce the route discovery frequency compared to the single path protocols [6]. These protocols maintain the alternate paths that have been already discovered, and use them to recover whenever the main path crashes. First, the path with the optimized hop count is used as a primary path. When this path fails, instead of launching another route discovery process, the alternate paths are used to continue packets transmission. This may reduce the number of routes discovery packets transmitted across the network to search alternative paths. Moreover, a higher number of alternate paths are provided whenever the node density in network raises [7].

These Multipath on-demand protocols minimize the number of new routes discovering process and eventually reduce the packets loss resulting a better packet delivery ratio and enhanced throughput; however, it is still suffering in high dynamic topology [8].

In literature, main works tries to enhance the performance of the AOMDV protocol, by exchanging packet to reveal nodes battery, node geographical coordinates and velocity. These information are used to estimate link failure or nodes life time [9]. However, the network status is very dynamic. The use of historical traffic in network to enhance the performance can give a better overview of the network performance and reduce the number of packet exchanged between nodes. In this paper we have used historical variation of nodes energy values to increase the nodes life time and reduce overhead. 
Taking in account the fact that Energy consumption of nodes depends mainly on the number of transmitted and received packets. We conclude that the node energy variation depends then on several factors such as the number of packet in the node's buffer, frequent route failure leading to increase control and data packets transmission, the number of control and data packet passing throw this node, etc. For example an increased energy variation of a specific node between two slots of time $t$ and $t+1$, may be justified by the increased packet flow passing by this node.

In our approach, we have used the historical values of nodes energy to compute energy variation rate. This information can reveal if there is an excessive consumption of energy. Multiples actions may increase the energy consumption such as an increased packet passing throw the node, increased route error packet transmission, etc.

The main objective of this work is to maximize nodes lifetime, reduce overhead, and to conserve energy consumption of the AOMDV protocol. The proposed scheme attempts to solve the above mentioned problem by relying on nodes with the minimal energy variation to root packets. This paper is organized as follows: Section 2 describes the related work, we explain how the AOMDV protocol performs and we give a detailed description of the principle procedures of the protocol in section 3. The mathematic model used to reveal energy variation rate during time and the implementation of the proposed approach are discussed in section 4. The performance parameters, results and analysis are detailed in section 5 . We conclude the paper and present the future perspectives.

\section{Related work}

\subsection{Ad Hoc On-demand, multipath distance vector routing protocol}

Several studies were conducted to reduce the link failure of the AOMDV protocol. Joshi et al. [6] implemented predictive pre-emptive AOMDV routing protocol for MANET. Authors used an energy threshold to predict a link failure in paths from source to destination, afterward, if the main path will probably crash, the source will pre-select another alternate path from the backup paths stored in its routing table. Simulation result shows reduced packet drop ratio and increased network throughput. However, the proposed protocol adds location information in the RREQ packet such as the velocity, position, and movement direction of the source. This information can lead to excessive consumption of energy using GPS devices, moreover an extra size of packet is added, which can cause an increased overhead.

Kalpana and Karthik [10] added a new mechanism to predict the link breakage in AOMDV based on signal strength. The proposed protocol is capable to determine if the quality of link will be improved (i.e. stable) or will be reduced (i.e. probability of failure) in order to anticipate link breakage and improve QoS. The proposed protocol predicts the future disconnection of an active route to provide another cached link before break occurs. When the signal quality is declining due to neighbour node remoteness, a discovery of two hops part of the path will be established and it will be used when disconnection happens. The simulation results shows that the proposed protocol PF_AODV provides better performance 
in terms of throughput, packet loss and delay. However in dense and dynamic networks, a discovery of paths to rescue two more hops can increase the network overhead.

Mallapur and Patil [11], implemented a "stable backbone-based multipath routing protocol (SBMRP)". The proposed routing protocol tries to rely on the best paths from source to destination by routing packets throw candidate nodes which have the lowest mobility movement changes, the higher power capacity, the higher link quality, and the signal strength. The efficiency of this protocol is shown with less dropped packets and increased number of successfully packet received.

Gupta and Prasad [3], presented a congestion management mechanism in AOMDV protocol. They proposed dynamic queuing to reduce the overhead and congestion in network. First, the sender will obtain all information of intermediate nodes (queue size, data rate, etc.) in paths from source to destination. An acknowledgement packet is used to indicate capability of intermediate nodes. If the load is not balanced, source node will alter rate of every path and estimate the load. Simulation results show reduced delay and management overhead.

Sonawale and Nema [7], showed that the historical flow of control packet such as RREQs, RREP and REER messages in the network can be used to reveal the accessibility of nodes. Once we have the accessibility feature of each node, only the "accessible" and "start" nodes are allowed to initiate a route discovery process. This results in a better use of network resources. The performance results show that this approach can reduce the routing overhead and improve the Packet delivery ratio. However, to compute accessibility information, Routing table entries are never deleted. This may be a problem in dense network size.

\subsection{Energy and network life time}

Authors Sivaraman and Karthikeyan [12], have implemented an "Energy Efficient and Bandwidth Aware On-demand Multipath routing protocol for Mobile Ad hoc Networks (EE-BWA-AOMDV)". The proposed routing protocol relies on the best paths basing on the minimal residual energy and available bandwidth. The path with the greatest available bandwidth as its primary path for transfer data. The efficiency of this protocol is quite impressive with less dropped packets, less energy consumption and increased packet delivery rate. However counting always on nodes with minimum energy and high bandwidth may exhausted rapidly the nodes battery in time.

Aqeel et al. [13], proposed an energy efficient optimized AOMDV Routing protocol which could consider energy conservation using using Particle Swarm Optimization (PSO). A fitness function is used to find the optimal path from source node to destination node to reduce the energy consumption in multipath routing. The distance of this route will be considered, the optimum route refers to the route that has the highest energy level and the less distance. Whenever an intermediate node receives a route reply packet, it computes its remaining energy and add it to the energy field in the route reply packet. If the sender receives multiple reply, it will choose the path with the maximum average energy per hop value. Simulation results show a better performance in terms of delivery rate, end delay, and throughput. 
An improved energy efficient AODV routing protocol for MANETs is proposed by Patil Annapurna et al. [14]. Authors used the evaluation of nodes energy values to forward the packets with the least drained nodes paths, making the network adaptive in nature. The protocol uses the concept of drain count into AODV protocol. Drain counts are calculated based on residual energy of nodes. Paths with the least drain count are used to route packets. Simulation results show a reduced overhead and an increased network life time. However, in high dynamic scenarios, AODV has better values of throughput. The proposed protocol generates a lot of control packets and an extra computation cost when calculating drain.

A life time maximization of the AOMDV protocol is proposed by Liu et al. [15]. Authors balanced the energy consumption to preserves the residual energy of nodes and increase the network life time. The basic idea is straightforward with the use of two parameters: the energy threshold and the coefficient alpha. These parameters are used to ensure the preservation of the node energy. The protocol performance shows an increased network life time and reduced energy consumption.

A comparative analysis of the E-AOMDV protocol with the MC-AOMDV protocol using multi-criteria multipath Routing is presented by Kaur and Singh [16]. The residual energy of nodes and distance is taken into account while making multiple paths from source to the destination, to preserve the energy consumption in mobile ad hoc networks. NS2 simulation results showed that MC-AOMDV is better in terms of overhead and throughput.

In [17], authors studied the energy consumption in Wireless sensor networks (WSN), and presented different works made to improve the duration of network lifetime. WSN is set of very small device, used to collect information. Most of the cases these networks are placed in difficult areas to reach, in which it is difficult to charge and replace nodes battery, so the energy conserving of WSN nodes is crucial. To prolong the life of the network, several routing approaches have been proposed. Authors demonstrated that ART-LEACH protocol was able to improve energy consumption and extend life of the network by $40 \%$ compared to LEACH protocol and IB-LEACH. Hence a new approach was proposed where the authors to transfer and select the $\mathrm{CH}$ (Cluster Head). The simulation results showed that the proposed approach reduce energy consumption and increase the duration of network life.

In [18], presented a survey of power saving and energy optimization techniques for wireless sensor networks. The authors discussed the wireless hardware structure of senor node, the characteristics and requirements of a wireless sensor node and the energy wastage given by electronic circuit. The authors showed that the electronic design that includes the right component for nodes is crucial to enhance the network performance. In the end, the authors compared between several MAC and routing protocols that have been designed to optimize the power consumption.

\section{AOMDV Routing Protocol Overview}

AOMDV "Ad Hoc On-demand, multipath distance vector routing protocol", is a special routing protocol designed for MANET environment. The key concept of the AOMDV 
protocol is to compute multiple loop-free paths, to switch to an alternate path if the main path the destination node crashes. AOMDV can use two approaches to find node-disjoint or link-disjoint paths [10]. A new route discovering process is initiated only after the failure of all disjoint paths.

In the Node disjoint path each intermediate node does not reject immediately the route request RREQ coming from the source node arriving via a different neighbour, the path is stored in the routing table of the intermediate node to mark a node disjoint path. However these route requests are not propagated, due the fact that broadcasting more than one RREQ may lead to have more than one path traversing the same intermediate node. When a route reply RREP is received, the node replies the source via the reverse disjoint path in its routing table. Note that path with the node disjointness is stricter then paths with the link disjointness. The link disjointness approach allows computing multiple paths passing by the same node, thus common nodes may be founded in paths from source to destination, when an intermediate node receives a RREP from the destination, and it replies the source via the link disjoint paths in its routing table.AOMDV uses advertised hop-count to ensure not getting any routing loops. Mainly there are three principle procedures in the AOMDV Routing Protocol:

\subsection{Send Route request}

When a source needs to deliver packets to a destination it first checks whether it has a valid pre-existing path to it in its routing table. If it is the case, it delivers the content directly .If not, it stores the current packets in a buffer (the packets are buffered in a FIFO "first in, first out" queue) and transmit them to the destination whenever a route is set [19]. Afterward, the source will trigger a new route discovery process in order to find a path to the destination, following these key action rules:

- After sending a Route Requests a timer is set, when the timer is expires without getting any response from the destination, a new route discovery process is initiated.

- $\quad$ The source is allowed to rebroadcast the RREQ packets for a maximum number, after that the source will stop sending route requests to prevent the increasing of the network overhead with an endless broadcast. Figure 1 shows the RREQ packet flood in network during route discovery.

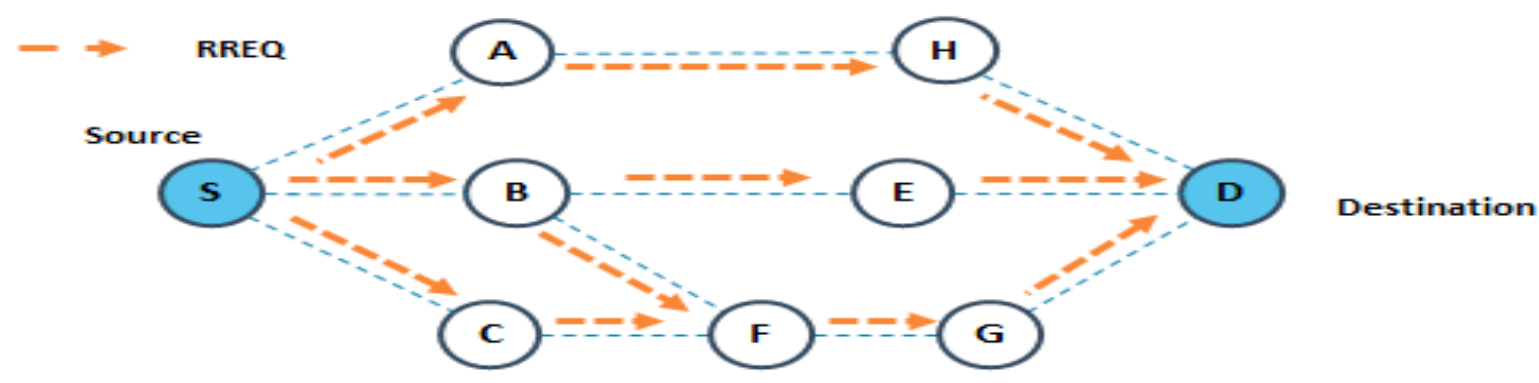

Figure 1. Route discovery packets flood in network. 
- The time to live value (TTL) is used to indicate the maximum hop count for packets to be forwarded. This value is set to a predefined value in the first transmission and increased to a greater value in the second retransmissions (to prevent putting more overhead). Retransmissions occur only if no replies are received.

\subsection{Receive, Forward and Reply a Route Request}

When a node receives a RREQ from an intermediate node it checks whether if this is the first time to receive RREQ from the source. If so, it will forward this RREQ packet else it will stop the propagation and drop the received RREQ packet. Secondly the current nodes checks if it is the first node towards destination, if so, it will set the field of the first hop of RREQ by its address.

A new route entry is created if the route entry of the source doesn't exists. In the case where, the source sequence number of the received packet is greater than the source sequence number in the routing table that already exists then : the node will update the source sequence number in the routing table, delete all old paths that already exist, create a reverse path to the new fresh path and set the hop count filed as the maximum hop count [20].

In the case where the source sequence number of the received packet is equal to the sequence number in the routing table that already exist then: the node checks if this new path already exists, if so, it will update the expiration time. If this path doesn't exist, and it's a new disjoint path (no path with the first hop as the last hop exists for this route entry), the node will add the new path to the routing table only if there is not too many paths that already exists for this destination, and the new path does not differ too much in length compared to existing paths. The packet is dropped if there is a path with the same first hop node and has less hop count than the received packet.

If the current node is the destination or have a valid path to it, it will send a route reply RREP else the node will forward the RREQ packet. When the intermediate node receives the RREP, it will add the destination to its routing table and create a reverse path to it, after that the intermediate nodes will backward the RREP until it reaches the source, hence multiple paths then are formed from source to destination. Figure 2 shows Route Reply packets flood in network and path establishment.

\begin{tabular}{|l|l|l|l|}
\hline \multicolumn{4}{|c|}{ Source route table } \\
\hline Dest & $\begin{array}{l}\text { Next } \\
\text { hop }\end{array}$ & $\begin{array}{l}\text { Hop } \\
\text { count }\end{array}$ & $\begin{array}{l}\text { Last } \\
\text { hop }\end{array}$ \\
\hline D & A & 3 & H \\
\hline D & B & 3 & E \\
\hline D & C & 4 & G \\
\hline
\end{tabular}

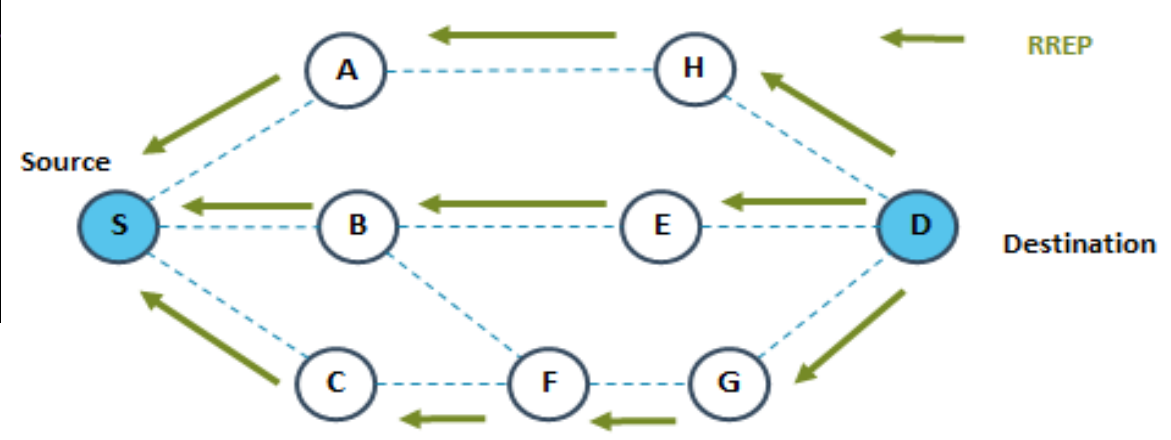

Figure 2. Route Reply flood in network and path establishment 


\section{Macrothink}

\subsection{Route Maintenance}

To maintain a fresh status of disjoint paths, Hello messages are broadcasted periodically between neighbours. When receiving these messages, the expire time of link with neighbours is extended. If no messages were received, it means that these neighbours are no longer reachable and probably a link failure within the path between source and destination has occurred [21].

Multiple reasons can cause links failure (network congestion, node mobility or power failure, etc. To recover from a link failure, a Route maintenance procedure is launched. The route maintenance consists of sending a Route Error message RERR backward to the source node. The source node selects another secondary path for packet transmission. The best available path among the alternative paths is chosen. When all the alternative paths fail, the source restarts the route discovery process again. Figure 3 shows the REER flood in network [22].

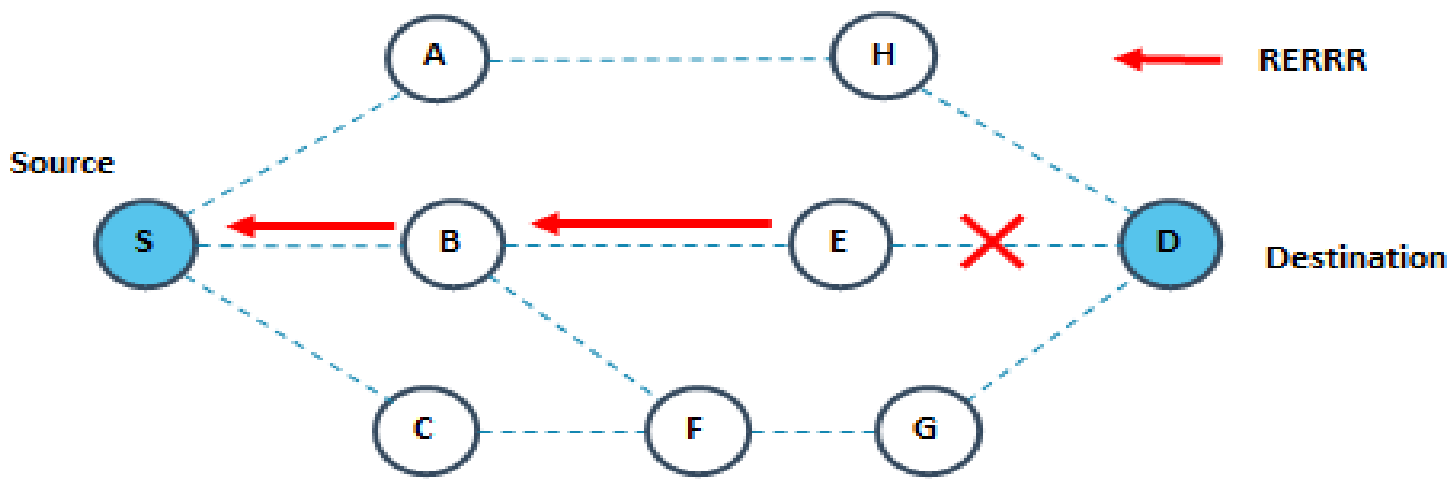

Figure 3. Route Reply flood in the network and path establishment

\section{Proposed Approach}

\subsection{Problem definition}

In this Section we describe the mathematical formulas used in this paper. Let the ad hoc wireless network be presented as graph $G(N, L)$, where $N$ represents nodes in network, and $L$ represents the unidirectional links between nodes.

Let $E g(n, t)$ be the energy of a node $n$ at time $t \quad / n \in N$;

Let $I$ be an interval of time;

Let $T p$ be the packet transmission power;

Let $S z$ (pckt) be the packet size;

Let $B w$ be the bandwidth;

Let $E g$ (pckt) be the energy required to transmit a packet, it is calculated as follow: 


$$
E g(p c k t)=\frac{T p \cdot S z(p c k t)}{B w} \quad .
$$

Let $F$ be a function of energy consumption in $I$;

Let $D p$ be the number of data packets;

Let $\mathrm{Ng}$ be the number of neighbours in which a hello messages are received each I;

Let $E p$ be the number of error messages;

Let $C p$ be the number of control packets such as RREQs.

The energy conception function $F$ is given by:

$$
F(D p, N g, E p, C p)=(D p+N g+C p+E p) \cdot E g(p c k t)
$$

The residual energy after one slot of time I is given by:

$$
E g(n, t+I)=E g(n, t)-F(D p, N g, E p, C p)
$$

The energy consumption between two slots of time is given as below:

$$
C=|E g(n, t+I)-E g(n, t)|
$$

The energy rate variation is given as follow:

$$
V=\frac{C}{E g(n, t)} \cdot 100
$$

Given two nodes $n 1$ and $n 2$, the node with an increased number of neighbouring packets, route failure and data transmission packets, will consume an increased energy during time, hence the energy variation in time will be increased. A pre-emptive approach to reduce load on node with excessive energy rate variation is discussed in the next session.

\subsection{Proposed scheme}

We have used the energy rate variation between two slots of time to detect all actions decreasing energy such as an increased routing packet transmission (Hello message, error packet and RREQ retransmission due to links failure). This may better reveal nodes with excessive energy consumption.

In each interval of time the node will calculate the variation rate of its energy. Hello time interval is chosen to calculate this information. Meanwhile, to maintain a fresh a status of the network state, in each hello interval of time, a hello message is broadcasted between nodes to detect broken links. We added new field called Pckt_var to the hello packet. In the table down below we present the notation used in algorithms: 
Table 1. Notation Description of Algorithms

\begin{tabular}{|c|c|}
\hline Notation & Description \\
\hline Htime_intv & Hello time interval \\
\hline Eng_(t) & Current energy of the node at $t$ \\
\hline $\mathrm{t}$ & Current time \\
\hline $\mathrm{V}$ & Energy variation rate \\
\hline Eng_th1 & Excessive consumption threshold \\
\hline Eng_th2 & Overload consumption threshold \\
\hline Pckt_var & Variation field added in packets \\
\hline Rt_entry & Route entry \\
\hline Rt_table & Routing table \\
\hline Ngb_nd & Neighbour node \\
\hline Int_nd & Intermediate node \\
\hline Id_Ngb & Neighbour Id \\
\hline $\mathrm{S}$ and $\mathrm{D}$ & Source and destination nodes \\
\hline Cp & Control packet \\
\hline Marked & $\begin{array}{l}\text { Field in a path record indicating overload consumption of } \\
\text { energy }\end{array}$ \\
\hline Buff_pckt & Packet buffer \\
\hline min_hp & Minimum hop count \\
\hline Htime_intv & Hello time interval \\
\hline Eng_(t) & Current energy of the node at $t$ \\
\hline $\mathrm{t}$ & Current time \\
\hline $\mathrm{V}$ & Energy variation rate \\
\hline Eng_th1 & Excessive consumption threshold \\
\hline Eng_th2 & Overload consumption threshold \\
\hline Pckt_var & Variation field added in packets \\
\hline Rt_entry & Route entry \\
\hline Rt_table & Routing table \\
\hline Ngb_nd & Neighbour node \\
\hline Int_nd & Intermediate node \\
\hline Id_Ngb & Neighbour Id \\
\hline S and D & Source and destination nodes \\
\hline Cp & Control packet \\
\hline Marked & $\begin{array}{l}\text { Field in a path record indicating overload consumption of } \\
\text { energy }\end{array}$ \\
\hline Buff_pckt & Packet buffer \\
\hline min_hp & Minimum hop count \\
\hline
\end{tabular}

In the case where the node has an overload energy consumption; other nodes will be notified when receiving the hello message. Algorithm 1 shows the energy rate variation computing as shown in (3) and (4). 


\section{Algorithm 1 : Energy Rate Variation Compute}

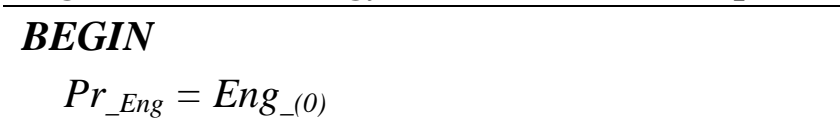

For each Htime_intv

$$
\begin{aligned}
& V=\left(\left(\text { Eng_( }(t)_{-}-r_{-E n g}\right) / P r_{-E n g}\right) * 100 \\
& P r_{-E n g}=E_{\text {Eng_( }}(t) \\
& \text { HELLO-> Pckt_var }=V
\end{aligned}
$$

If there is an active route then

Send Hello

\section{End for each}

\section{END}

The node keep in cache its energy rate variation. Two threshold Eng_th1 and Eng_th2 has been fixed based on the simulation results where Eng_th2 < Eng_th1. These threshold were set to detect excessive and overload energy consumption. Each RREQ or RREP received is compared with Eng_th1, in the case where the energy rate variation of a specific node exceeds Eng_th1 (excessive energy consumption), the node will no longer participate in the future discovering of routes and ignore discovering packets. In the contrary case, the node adds its energy rate variation in the packet and continue the packet forward.

The node will notify its neighbours constantly about its energy rate variation. When the RREQ, RREP or Hello packet arrives to neighbours, they will compare it with Eng_th2 (overload energy consumption), if it exceeds Eng_th2, the neighbours will not try to exhaust this node and rely on alternate paths with reasonable energy consumption if they exist. A new field called Pckt_Var is added to route request RREQ, route reply RREP and Hello packets as shown in Figure 4.

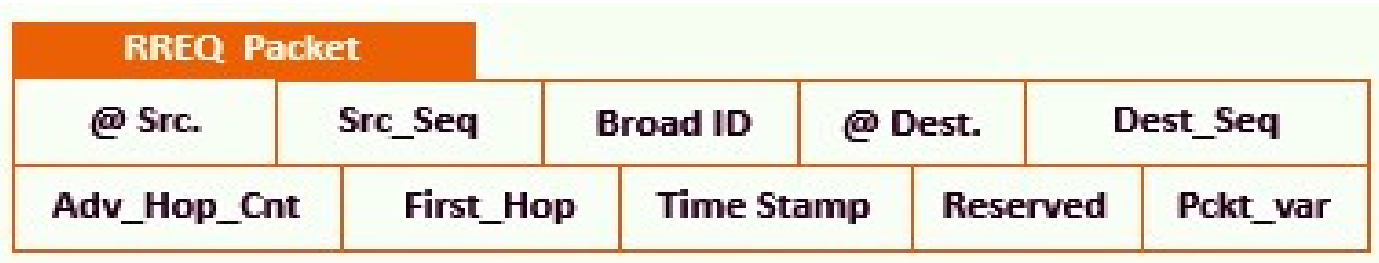

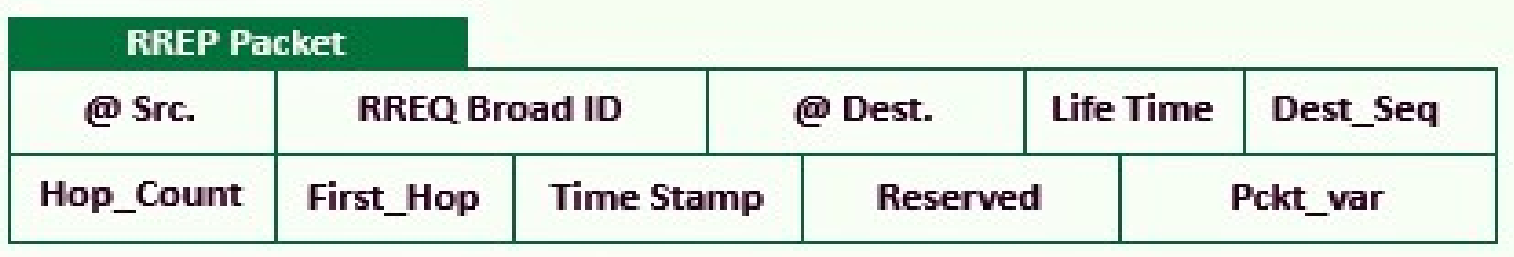

\begin{tabular}{|c|c|c|c|}
\hline \multicolumn{2}{|c|}{ Hello Packet } \\
\hline @ Src. & @ Dest. & Dest_Seq & Time Stamp \\
\hline Hop_Count & Life Time & Reserved & Pckt_var \\
\hline
\end{tabular}

Figure 4. AOMDV packets format 
The notation used to descript packets fields are presented in the table down below:

Table 2. Notation Description of Packets

\begin{tabular}{|l|l|}
\hline Parameter & Value \\
\hline \hline @ Src, @Dest & Addresses of source and destination nodes. \\
\hline Src_seq, Dest_Seq & Sequence number of source and destination nodes. \\
\hline Broadcast_Id & Id of broadcast RREQ packet. \\
\hline Adv_Hop_Cnt & Advertise Hop count. \\
\hline Life Time & Contains life time of the packet \\
\hline First_Hop & First hop in path from source to destination. \\
\hline Hop_count & Hop count between source and destination. \\
\hline Reserved & Reserved field in packet. \\
\hline Timestamp & Used to compute route discovery latency when \\
& corresponding RREQ is sent. \\
\hline Pckt_var & Energy rate variation in packets. \\
\hline
\end{tabular}

The algorithm 2 below shows the detail description of our proposed approach. First the sender broadcasts a RREQ to find a route to the destination. When the intermediate node Int_nd receives the control packet $C p$ (Hello, or route request packet RREQ, or route reply message RREP), it checks whether it can be forwarded. The control packet is forwarded just in the case where the energy variation of the node $V\left(I n t \_n d\right)$ doesn't exceed Eng_th1. In the contrary case, the node will not participate in route discovering and will drop the RREQ packet due its excessive consumption of energy. This may exclude nodes with an excessive energy consumption from route discovering.

If the node does note consume excessively the energy, the intermediate node compare the energy variation of the neighbour $V\left(N g b \_n d\right)$ that has send the RREQ with Eng_th2, if $V$ $\left(N g b \_n d\right)>$ Eng_th2, that's means that the node has an overload consumption of energy. The reverse path to the source is added and marked to indicate overload consumption (a field named marked is added to the path, it is set to be true if overload energy consumption is detected: New_path.marked $=$ true). Eng_th2 is set to reduce load on nodes with an overload energy consumption.

In the end, the intermediate node adds it energy variation rate to the packet and continue the packet forwarding. The packet is forwarded until it reaches the destination. The destination reply with a RREP. When intermediate nodes receives the route reply packet, it will repeat the same procedure of energy variation comparison with Eng_th2 and Eng_th1 to exclude nodes with excessive energy consumption from route discovering and reduce load and packet transmission frequency on nodes with an overload energy consumption.

When a node receives a hello packet ( $C p==$ Hello) from a neighbour it checks if it has an overload energy consumption, if so, all paths where this neighbour is the next hop to reach a specific destination is marked. These paths are exempted from packet transmission if there were alternate paths with reasonable energy consumption. 
Algorithm 2: Packets Control Receive and Treatment

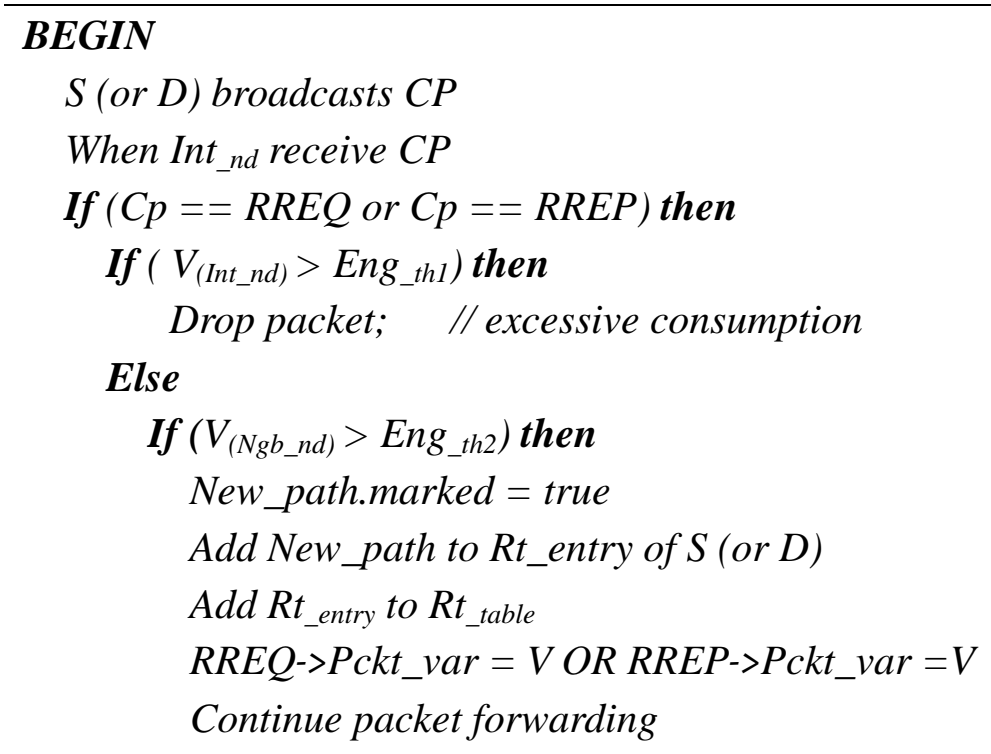

End If

End If

Else

If (Cp $==$ Hello) then

If $\left(V_{\left(N g b \_n d\right)}>\right.$ Eng_th2 $)$ then

For each $R t_{-}$entry in $R t_{-}$table

For each path in $R t_{-}$entry

If (path->next_hop $==I d_{-} N g b$ ) then

Path->marked $=$ true

End For each

End For each

End If

End If

END

First the node will check if the packet buffer Buff_pckt is not empty and there is packets to be sent, the node will search for the route entry of each packet, afterward it will search for the optimal paths in hop count where the energy consumption is reasonable. Path2 receives the pointer of the selected path. The node will also search the optimal path from paths with overloaded consumption energy (path->marked == true), Path1 receives the pointer of the selected path from paths set.

Finally, the algorithm will return the optimal hop count path from the paths with reasonable consumption, if there is no path with reasonable consumption energy where Path2 is null, the algorithm return the optimal path from overloaded consumption paths (Path 1).

The paths with the min hop count and which are not excessive in energy consumption are chosen. Therefore nodes with overload energy consumption due to frequent packet transmission will avoid battery exhaustion and be alive for much longer time. This may 
reduce also the collision and the route error RRER packet resulting in a better packet delivery ratio and decreased link failure. The algorithm 3 describes the procedure.

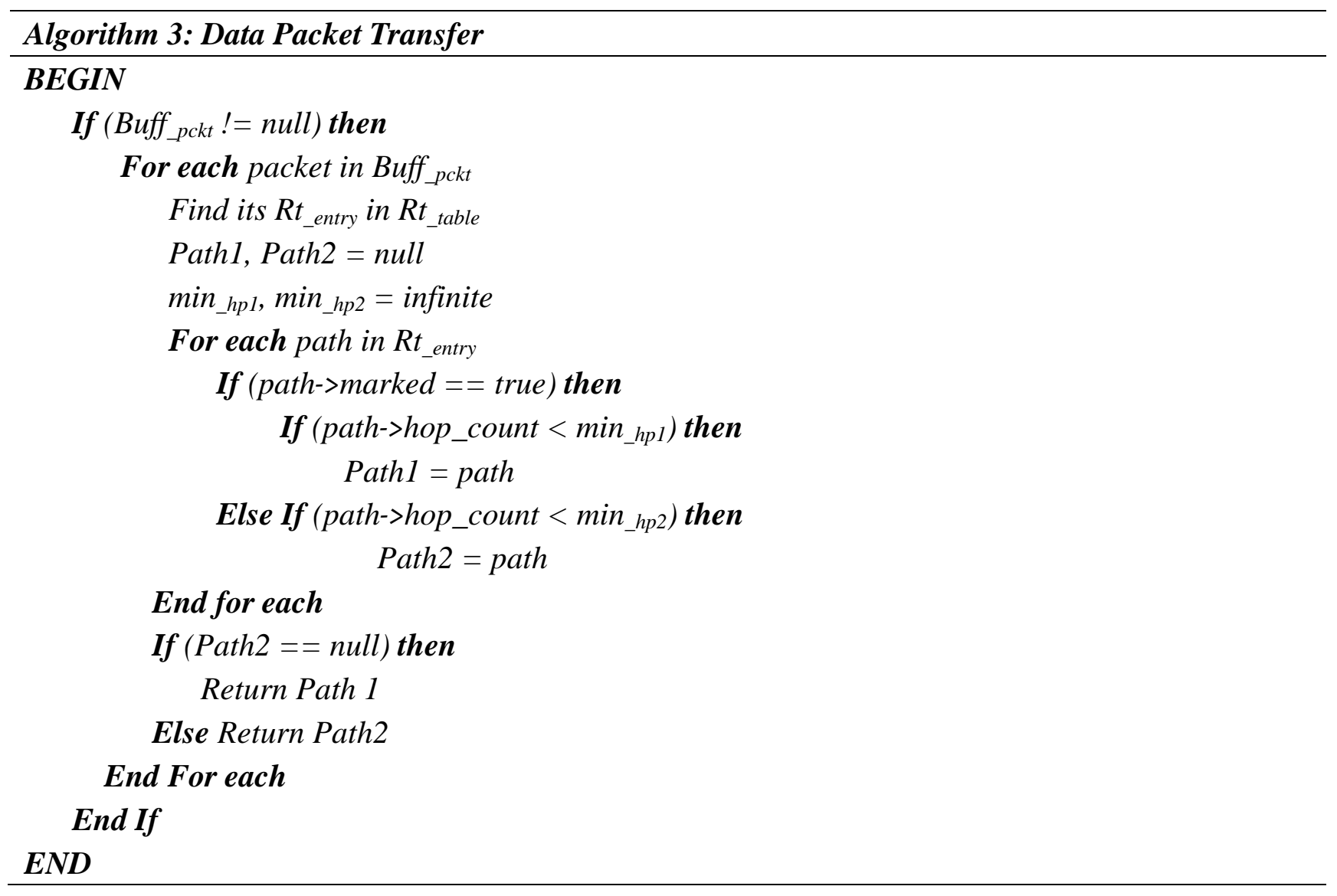

The steps of our proposed algorithm are described as follow:

1. Each node calculates its rate variation and keep it in cache. The source node send a RREQ to find destination.

2. Intermediate nodes receives the RREQ, if the energy rate variation of the current node is lower than the excessive threshold of consumption, the node will continue the packet forward and adds it's energy variation to the packet else the packet is dropped.

3. The destination receives the RREQ and sends a route reply RREP to the source.

4. Intermediate nodes receive RREP, adds its energy rate variation and continue packet forward if the consumption is not excessive.

5. If paths are established, the source counts on paths with a reasonable consumption energy to route packets.

6. When a node is notified by a hello message that its neighbour has overloaded consumption, the node will try to route packet by using another alternate route. 


\section{1l Macrothink}

\section{Simulation and Results}

Experimenting the performance of the network communication protocols under real environment conditions is crucial to evaluate their effectiveness.

Due to the high cost of these experimentation, we make call of the analytic and simulation methods. Both analytical and simulation methods are modelling approaches which aim at providing an idea of system performance, in different conditions. To run our simulations we used the well-known open source network simulator NS2.

NS2 is designed specifically for research in computer communication networks. It contains modules for numerous network components such as routing, transport layer protocol, and application. To investigate network performance, researchers can simply use an easy scripting language to configure a network and observe results generated by NS2. Figure 5 shows the basic architecture of NS2 [23].

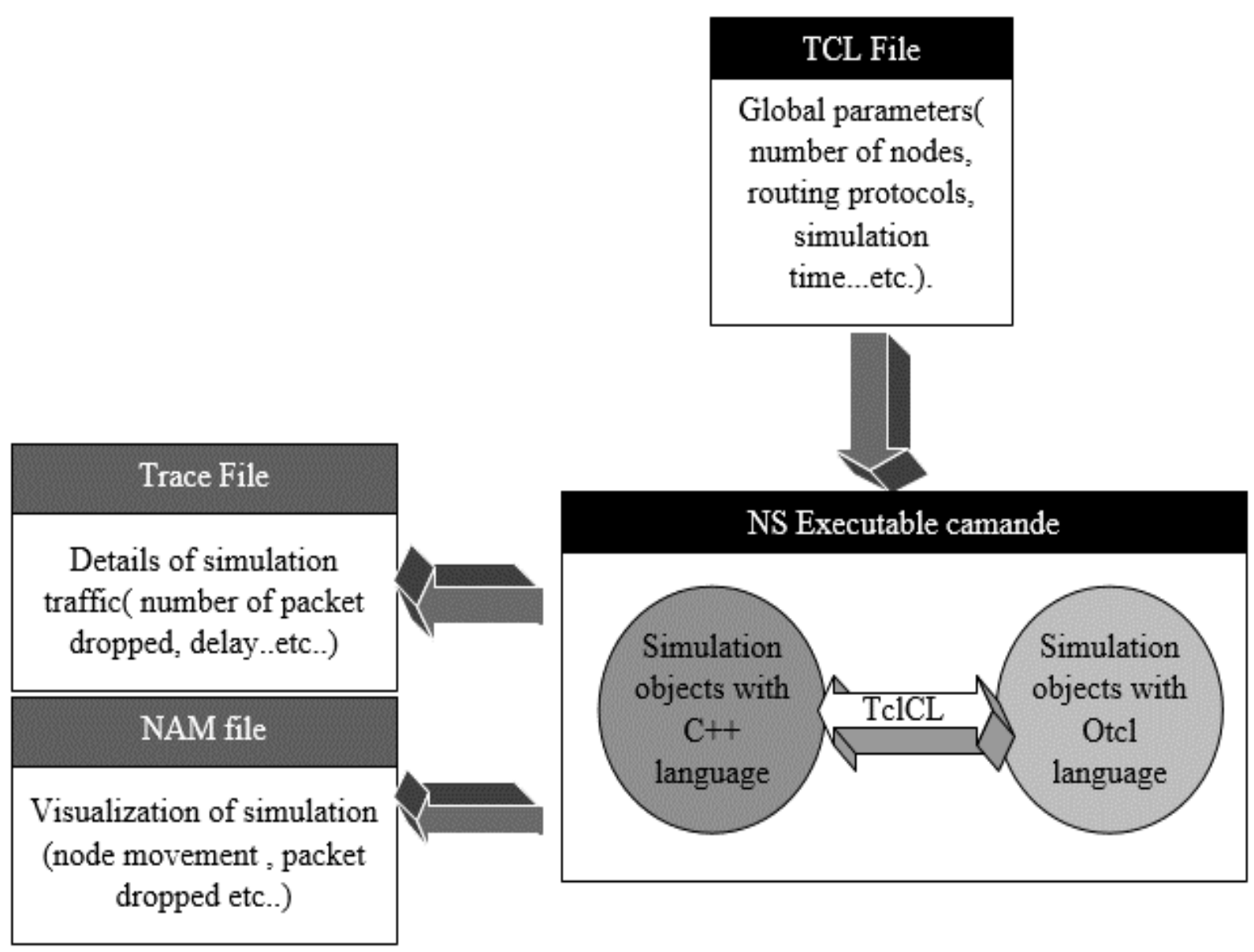

Figure 5. Network simulator NS2 architecture

A comparison study is done with our algorithm called pre-emptive conserving life time AOMDV based on energy variation (PCEV_AOMDV) and the basic AOMDV protocol in the presence of mobility and route failure. The simulation parameters are summarized in the table below: 
Table 3. Simulation Parameters.

\begin{tabular}{|l|l|}
\hline \multicolumn{1}{|c|}{ Parameter } & \multicolumn{1}{c|}{ Values } \\
\hline Routing protocol & AOMDV, PCEV_AOMDV \\
\hline Terrain size & $1500 x 1500$ \\
\hline Nodes & 100 \\
\hline Pause time & $0,2,4,6,8,10$ \\
\hline Communication pattern & 40 \\
\hline Connexion Rate & 8 packets/s \\
\hline Simulation Times & $200,250,300,350,400 \quad, 450,500,550,600$ \\
\hline Reception energy & $1 \mathrm{w}$ \\
\hline Transmission energy & $1 \mathrm{w}$ \\
\hline Initial energy & $100 \mathrm{j}$ \\
\hline
\end{tabular}

We have used several metrics to evaluate our approach:

- The Packet Delivery Ratio: the number of pockets delivered successfully.

- The routing overhead: the number of control packet broadcasted in network divided by the total number of packet sent from source to destination.

- The total energy consumption by nodes: this value is calculated in the end of simulation by the summation of the initial energy minus the current energy of nodes

- Alive nodes: the number of alive nodes by the end of simulation.

Simulation output is visualized in Figure 6.

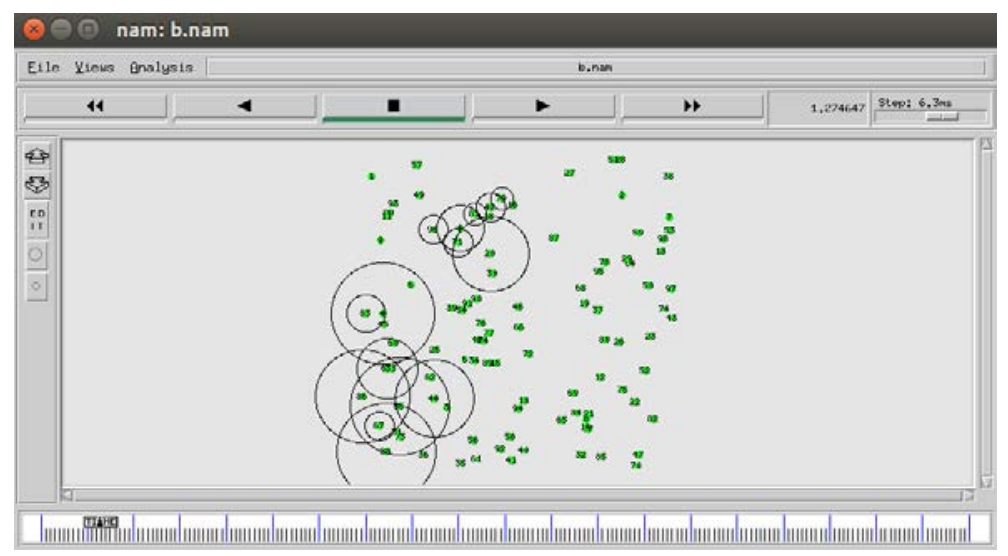

Figure 6. Simulation output

Avoiding sending packet with nodes that consume more than $0.5 \%$ of their energy (overload energy consumption) and exclude the nodes with excessive energy consumption more than $10 \%$, may reduce the probability of nodes death, this may avoid causing the launch of route error and route request packet in network leading to save nodes battery and increase the life time of nodes. In Figure 7, a better performance of our approach comparing to AOMDV is noticed in terms of alive nodes varying the time of the simulation. The best performance is noticed when the time of simulation is 350 seconds, the number of alive nodes is enhanced with $30 \%$ compared to the basic AOMDV protocol. 
Due to frequent link failure, rebroadcasting the RREQ packets to find routes from source to destination may increase the congestion of the network. With our proposed approach the network overhead is reduced by reducing the number of RRER and RERR broadcasted due to dead nodes. Figure 8 shows a reduced overhead in network using the proposed protocol PCEV_AOMDV varying the pause time. When pause time is 4 seconds, the overhead is reduced with $16 \%$.

Avoiding the exhaust of overload nodes by the rebroadcast of RREQ and RRER packets due to nodes dead reduce the energy consumption using our protocol. Figure 9 shows the consumption energy of AOMDV comparing to the proposed approach. When the pause time is 0 , the energy consumption is reduced from $7049 \mathrm{~J}$ to $6678 \mathrm{~J}$.

Figure 10 shows packet delivery ratio of the basic AOMDV comparing with our proposed approach varying pause time. It is obvious that PCEV_AOMDV has a reduced packet dropped ratio than AOMDV routing protocol due the fact that PCEV_AOMDV avoid to route packet with paths with overload nodes, which can extend the path life time and avoid link failure. Thus, the ratio of the packet received successfully raises. When the pause time is 0 the packet delivery ratio increases with $4 \%$.

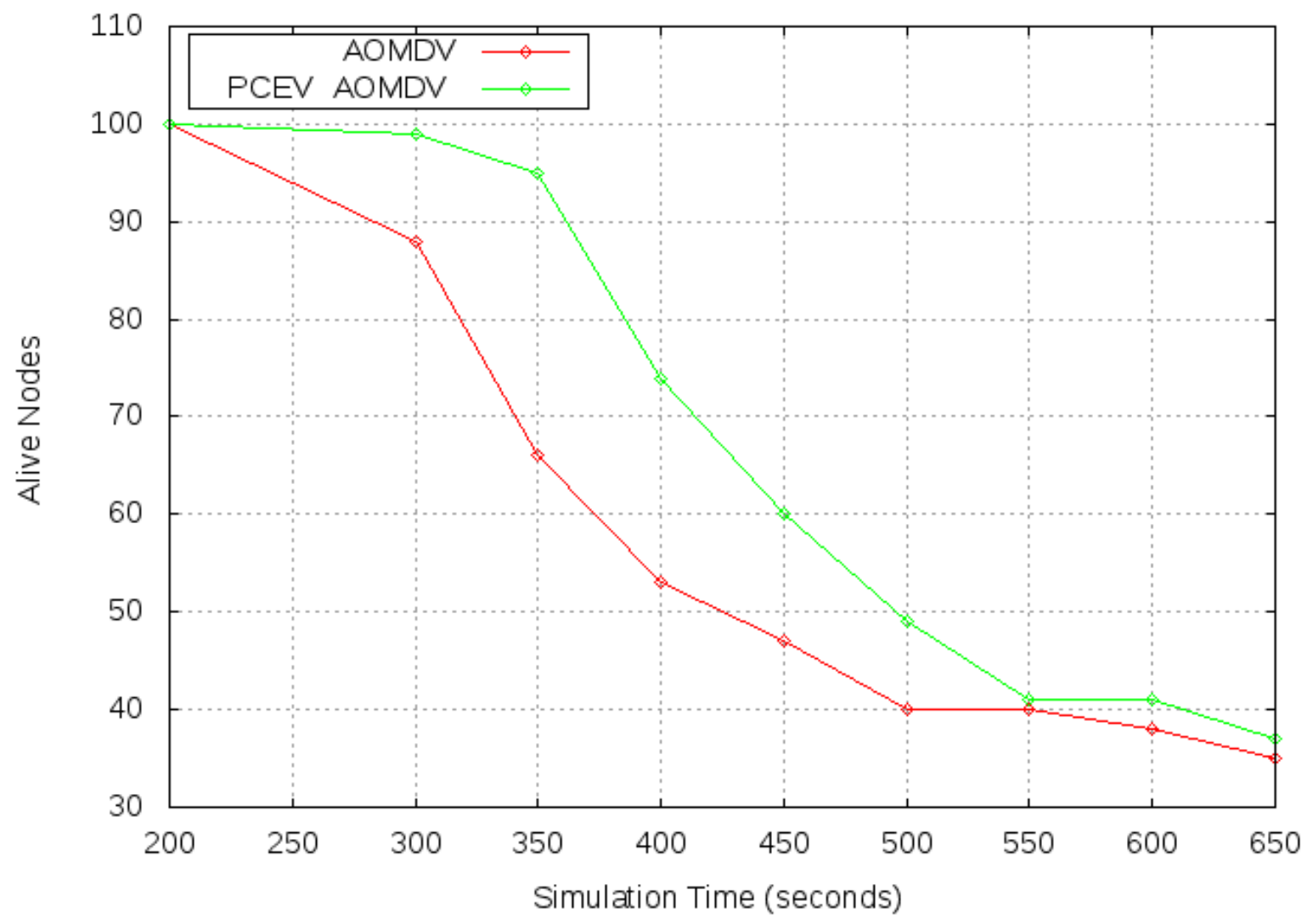

Figure 7. Number of alive nodes 


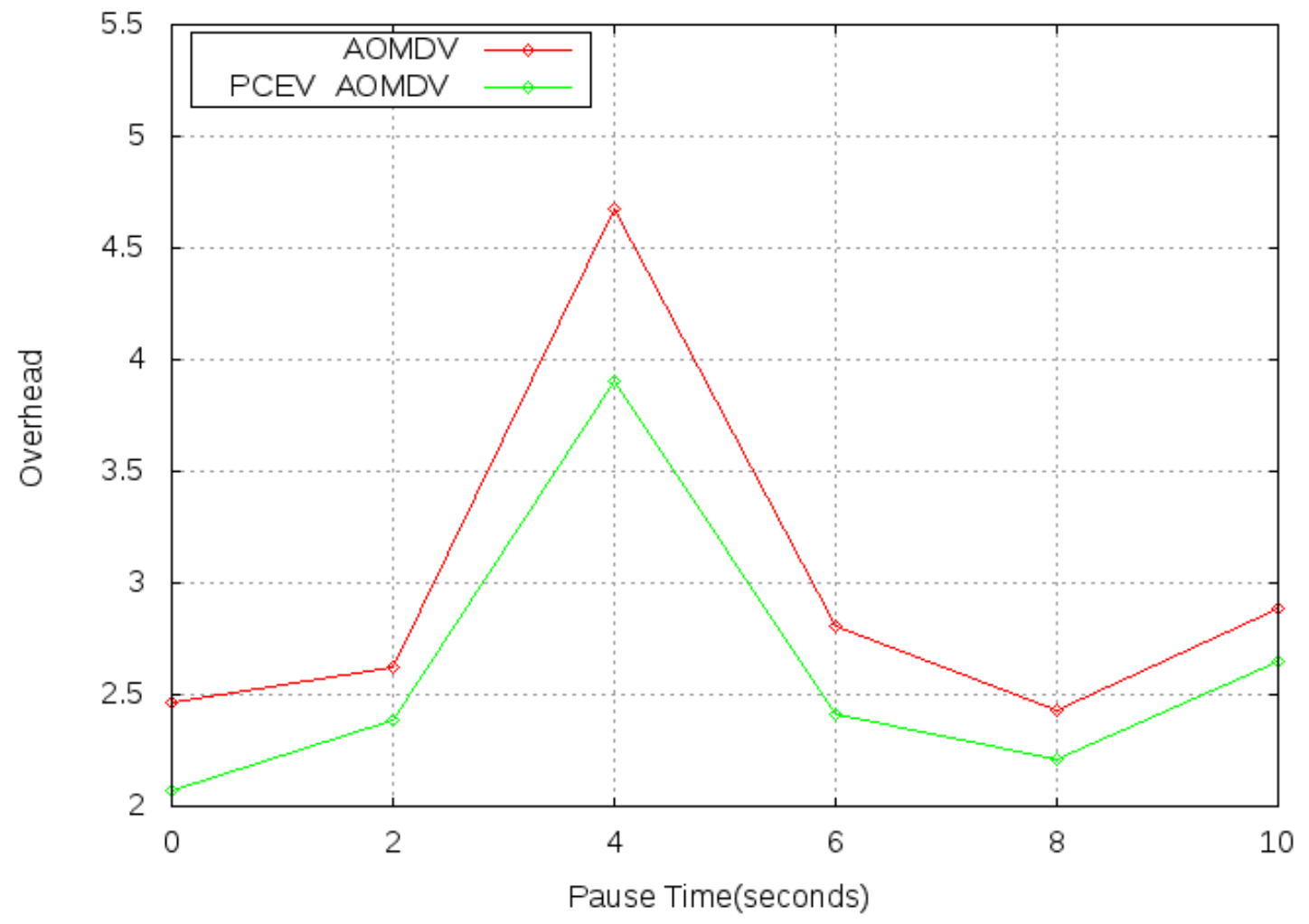

Figure 8. Overhead management

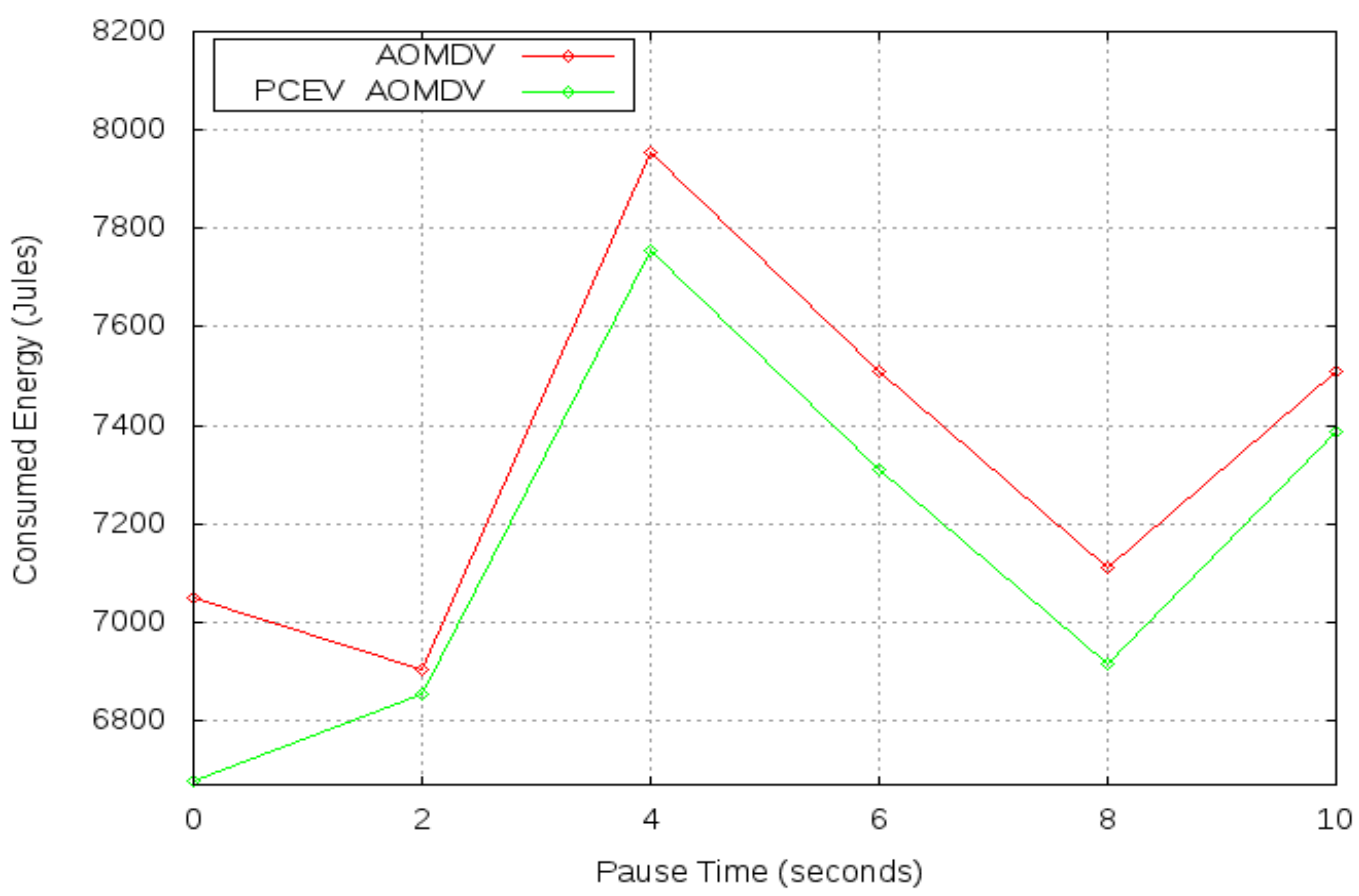

Figure 9. Consumed Energy 


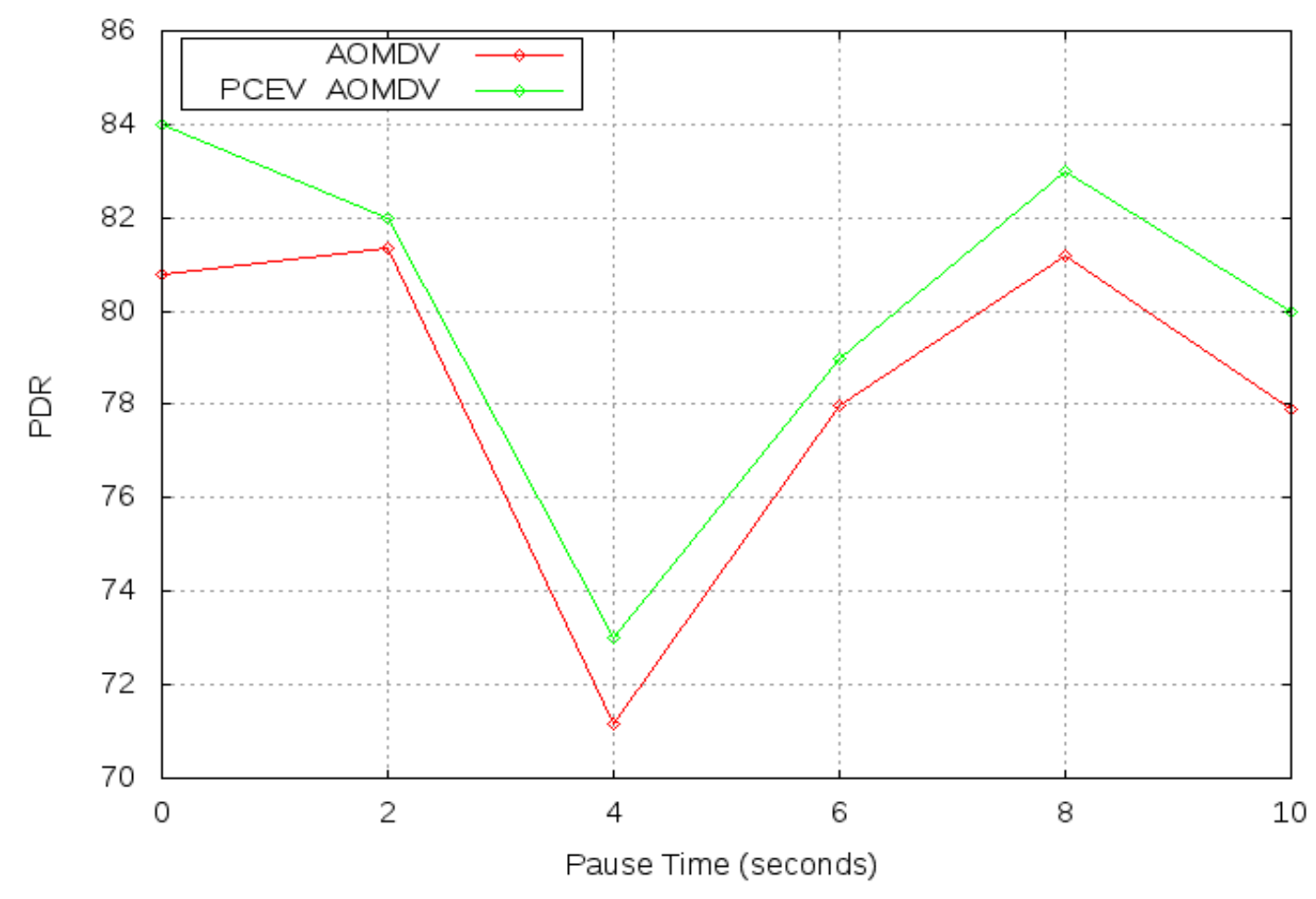

Figure 10. Packet Delivery ratio

\section{Conclusion and Perspectives}

AOMDV protocol is efficient and may reduce significantly the overhead, packet lost and increase the packet delivery ratio comparing to AODV. However, it is still suffering in high dynamic topology. In this paper, a pre-emptive approach based on the energy rate variation of nodes is used to reduce the number of dead nodes. The proposed protocol PCEV_AOMDV detect overload nodes by using historical information of energy consumption and avoid sending packet throw them. Our approach have been validated throw a detailed simulation study using the well-known NS2 simulator. Several performance metrics are used to compare PCEV_AOMDV with the standard AOMVD. Simulation results show reduced number of dead nodes, reduced overhead and energy consumption and an enhanced packet delivery ratio.

As a future work, there are several scenarios that could be implemented with this study to increase nodes life time, reduce overhead and energy consumption. The cross-layer coordination and communication between the different Ad hoc network layers to improve the QoS parameter using historical information can give better network performance to overcome rapidly and effectively to congestion and link failure.

Acknowledgement: This work has also been partially supported by European Union through the ERANETMED (Euromediterranean Cooperation through ERANET joint activities and beyond) project ERANETMED3-227 SMARTWATIR. 


\section{References}

[1] Sambasivam P. and Murthy A., (2004) 'Dynamically Adaptive Multipath Routing based on AODV’, Mediterranean Ad Hoc Networking Workshop.

[2] Philomina S. and Ramesh R., (2017) 'TRR-AODV Based Performance Enhancement in Mobile Ad Hoc Network', Journal of Advanced Research in Dynamical and Control Systems, vol. 9, no. 1, pp. 35-40.

[3] Gupta S. and Prasad G., (2016) 'Enhanced Load Balancing and Delay Constraint AOMDV Routing In MANET', Symp. Colossal Data Anal. Networking. https:// doi.org/10.1109/CDAN.2016.7570871.

[4] Kaushik G. and Goyal S., (2013) 'An Clustering Based AODV Approach for MANET', International Conference on Emerging Trends in Engineering and Technology. https:// doi.org/10.5120/8122-1751.

[5] Santhi S. and Sudha Sadasivam G., (2014) 'Enhanced AOMDV for energy conservation in hybrid ad hoc networks', International Journal of Information and Communication Technology, vol. 6, no.2 pp. 122 - 141. https://doi.org/10.1504/IJICT.2014.060393.

[6] Joshi V., Biradar M., and Jeevangi S., (2013) 'Predictive Pre-emptive Ad-hoc On Demand Multipath Distance Vector Routing Protocol for MANET', International Journal of Engineering and Innovation Technology, vol. 3, no. 6, pp. 280-287.

[7] Sonawale T. A. and Nema S., (2012) 'Performance Comparison with Accessibility Predicion and Link Breakage Prediction in MANETS', International Journal of Computer and Communication Technology, vol. 5, no. 1, pp. 13-19.

[8] Kokilamani M. and Karthikeyan E., (2017) 'A novel optimal path selection strategy in MANET using energy awareness', International Journal of Mobile Network Design and Innovation $2017 \quad$ - Vol. 7, No.3/4 pp. 129 - $139 . \quad$ https://doi.org/ 10.1504/IJMNDI.2017.089284.

[9] Periyasamy, P. and Karthikeyan E., (2017) 'Link reliable energy efficient AOMDV routing protocol for mobile ad hoc networks', International Journal of Ad Hoc and Ubiquitous Computing, vol. 26, no.2 pp. 92 - 103. https://doi.org/ 10.1504/IJAHUC.2017.086261.

[10] Kalpana V. and Karthik S., (2017) 'Link Breakage Prediction in AOMDV Protocol to Improve Quality of Service in MANET', International Journal of Innovative Research in Computer and Communication Engineering, vol. 5, no. 2, pp. 1970-1977. https://doi.org/10.15680/IJIRCCE.2017. 0502163

[11] Mallapur S. V. and Patil S. R., (2013) 'Stable Backbone Based Multipath routing protocol for Mobile Ad-Hoc Networks', Proc. IEEE Int. Conf. Circuit, Power Comput. Technol. https://doi.org/10.1109/ICCPCT.2013.6528991.

[12] Sivaraman T. and Karthikeyan E., (2016) 'EE-BWA-AOMDV: Energy Efficient and 
Bandwidth Aware On-demand Multipath Routing protocol for Mobile Ad hoc Networks', International Journal of Computer Application, Vol. 6, no. 2, pp 2250-1797.

[13] Aqeel T., Raed A., Mueen Uddin, Maha A. and Tanzila S., (2017) 'Energy Efficient Multipath Routing Protocol for Mobile Ad-Hoc Network Using the Fitness Function', IEEE Access, vol. 5, pp. https://doi.org/10369-10381.10.1109/ACCESS.2017.2707537.

[14] Patil Annapurna P., Varsha Chandan B., Aparna S., Greeshma R. and Akshatha H P., (2014) 'An Improved Energy Efficient AODV Routing Protocol for MANETs', Wireless and Optical Communications Networks Conference. https://doi.org/10.1109/WOCN.2014.6923063.

[15] Liu J. and Chen J. and Kuo Y., (2014) 'Multipath Routing Protocol for Networks Lifetime Maximization in Ad-Hoc Networks', Wireless and Optical Communications Networks Conference. https://doi.org/10.1109/WICOM.2009.5305828.

[16] Kaur N. and Singh T., (2015) 'Comparative Analysis of E-AOMDV and MC-AOMDV using Multi-criteria Multipath Routing', International Journal of Computer Applications vol. 114, no. 9, pp. $0975-8887$.

[17] Ridha A., (2016) 'Consumption of Energy and Routing Protocols in Wireless Sensor Network', Network Protocols and Algorithms, vol 8, no 3 , pp. 76-87. https://doi.org/ 10.5296/npa.v8i3.10257

[18] Sendra S., Lloret J., García M., and Toledo JF., (2011) 'Power saving and energy optimization techniques for wireless sensor neworks', International Journal of communications Vol. 6, no. 6, pp 439-459. https://doi.org/ 10.4304/jcm.6.6.439-459.

[19] Punde J., Pissinou N., Makki K., (2003) 'On Quality Of Service Routing in Ad-Hoc Networks*', Local Computer Networks Conference. https://doi.org/10.1109 /LCN.2003.1243138.

[20] Diwakar B. and Krishna K., (2015) 'congestion aware multi-path routing protocol for mobile ad-hoc networks', International Journal of Convergence Computing, vol. 1, no.3/4 , pp. 217 - 231. https://doi.org/10.1504/IJCONVC.2015.076028.

[21] Tyagi S., Som S., Ranab Q.P., (2016) ‘A Reliability Based Variant of AODV in MANETs: Proposal, Analysis and Comparison’, Procedia Computer Science Journal, vol. 79, pp. 903-911. https://doi.org/10.1016/j.procs.2016.03.112.

[22] Vinay R., Mitul Y., Sandeep K.,(2017) 'Energy consumption pattern and scalability of routing protocols for heterogeneous MANETs', International Journal of Communication Networks and Distributed Systems 2017 - Vol. 18, No.1 pp. 83 - 109. https://doi.org/10.1504/IJCNDS.2017.081445.

[23] T. Issariyakul and E. Hossain, Introduction to Network Simulator NS2, Springer 2008. https://doi.org/ 10.1007/978-0-387-71760-9. 


\section{Copyright Disclaimer}

Copyright reserved by the author(s).

This article is an open-access article distributed under the terms and conditions of the Creative Commons Attribution license (http://creativecommons.org/licenses/by/3.0/). 